\title{
Running on a treadmill: dynamic inhibition of APC/C by the spindle checkpoint
}

\author{
Laura A Díaz-Martínez* and Hongtao Yu
}

\author{
Address: Department of Pharmacology, University of Texas Southwestern Medical Center, 6001 Forest Park Road, Dallas, TX 75390-9041, USA \\ Email: Laura A Díaz-Martínez* - laura.diaz-martinez@utsouthwestern.edu; Hongtao Yu - hongtao.yu@utsouthwestern.edu \\ * Corresponding author
}

Published: 24 July 2007

Cell Division 2007, 2:23 doi:10.1186/1747-1028-2-23

This article is available from: http://www.celldiv.com/content/2/1/23

(C) 2007 Díaz-Martínez and Yu; licensee BioMed Central Ltd.

This is an Open Access article distributed under the terms of the Creative Commons Attribution License (http://creativecommons.org/licenses/by/2.0), which permits unrestricted use, distribution, and reproduction in any medium, provided the original work is properly cited.
Received: 10 July 2007

Accepted: 24 July 2007

\begin{abstract}
During mitosis, the genome duplicated during S-phase is synchronously and accurately segregated to the two daughter cells. The spindle checkpoint prevents premature sister-chromatid separation and mitotic exit. The anaphase-promoting complex/cyclosome (APC/C) is a key target of the spindle checkpoint. Upon checkpoint activation, the mitotic checkpoint complex (MCC) containing Mad2, Bub3, Mad3/BubRI and Cdc20 inhibits APC/C. Two independent studies in budding yeast have now shed light on the mechanism by which MCC inhibits APC/C. These studies indicate that Mad3 binds to the mitotic activator of APC/C Cdc20 using peptide motifs commonly found in APC/ $\mathrm{C}$ substrates and thus competes with $\mathrm{APC} / \mathrm{C}$ substrates for $\mathrm{APC} / \mathrm{CCdc}^{\mathrm{C} 20}$ binding. In addition, Mad3 binding to APC/CCdc20 induces Cdc20 ubiquitination by APC/C, leading to the dissociation of MCC. Meanwhile, two other studies have shown that a deubiquitinating enzyme is required for the spindle checkpoint whereas APC/C-dependent ubiquitination is needed for checkpoint inactivation. Collectively, these studies suggest a dynamic model for APC/CCdc20 regulation by $M C C$ in which APC/C- and Mad3-dependent ubiquitination of Cdc20 constitutes a self-regulated switch that rapidly inactivates the spindle checkpoint upon correct chromosome attachment.
\end{abstract}

\section{Background}

Accurate chromosome segregation is the key event of mitosis. Errors in this process result in aneuploidy and genome instability, which contributes to cancer progression [1-4]. Mitotic chromosomes consist of pairs of sister chromatids that separate at the onset of anaphase. Sisterchromatid cohesion keeps sister chromatids together from the very moment of chromosome duplication until their separation. At metaphase, sister kinetochores are attached to microtubules emanating from opposite poles, a process referred to as amphitelic attachment or bi-orientation. A multisubunit ubiquitin ligase called the anaphase-promoting complex or cyclosome (APC/C) in conjunction with its mitotic activator Cdc20 then medi- ates the degradation of cyclin $\mathrm{B}$ and securin, allowing the activation of separase, cleavage of cohesin, and equal partition of sister chromatids into the two daughter cells $[5,6]$. Because microtubule attachment to kinetochores occurs stochastically, improper kinetochore-microtubule attachments, such as syntelic (sister kinetochores attach to microtubules from the same pole), monotelic (only one sister kinetochore attached), and merotelic attachments (a kinetochore attaches to microtubules from both poles), can form during mitosis $[7,8]$. These improper attachments ought to be corrected prior to sister-chromatid separation. Cells use a control mechanism termed the spindle checkpoint to ensure that all chromosomes are properly 
attached before initiating chromosome segregation $[9,10]$.

The spindle checkpoint monitors kinetochore-microtubule attachment and possibly inter-kinetochore tension generated by amphitelic attachments [11,12]. The unattached kinetochores are thought to produce diffusible checkpoint signals that inhibit $\mathrm{APC} / \mathrm{C}^{\mathrm{Cdc} 20}$ and block sister-chromatid separation $[13,14]$. An important checkpoint inhibitor of $\mathrm{APC} / \mathrm{C}$ is the mitotic checkpoint complex that contains Mad2, Cdc20, Bub3 and BubR1 (Mad3 in budding yeast) [15], although it is presently unclear whether MCC constitutes the diffusible checkpoint signal and whether MCC only forms at the kinetochores [16-19]. Here, we review recent advances in our understanding of $\mathrm{APC} / \mathrm{C}$ regulation by the mitotic checkpoint complex.

\section{APC/C Regulation}

$\mathrm{APC} / \mathrm{C}$ is the only known molecular target of the spindle checkpoint, although there is evidence to suggest that other checkpoint targets might exist $[20,21]$. APC/Cmediated ubiquitination leads to the degradation of cyclin $B$ and securin $[5,21,22]$, allowing efficient sister chromatid separation and mitotic exit. The spindle checkpoint inhibits APC/C and prevents sister-chromatid separation and mitotic exit until all sister chormatids achieve bi-orientation [3,21-25].

In addition to its involvement in anaphase onset and mitotic exit, APC/C regulates other cell cycle events, such as the G1/S transition [26,27] and initiation of DNA replication $[28,29]$. A growing number of APC/C regulators are required for the precise regulation of $\mathrm{APC} / \mathrm{C}$ activity during different phases of the cell cycle [5,30-32]. These APC/C regulators can be divided into three categories: (i) APC/C activators, such as Cdc20 and Cdh1, which contribute to substrate recognition and specificity of APC/C [33-39]. (ii) Enzymatic regulators that post-translationally modify core APC/C subunits or its activators [31,4048]. (iii) APC/C inhibitors, such as MCC $[15,49,50]$ and Emi1 $[51,52]$ that regulate APC/C through direct binding to APC/C or Cdc20 or both.

\section{MCC, a key checkpoint inhibitor of APC/CCdc20}

The first identified checkpoint inhibitor of APC/C was Mad2 [53], which inhibits APC/C through direct binding to Cdc20 [54,55]. The Mad2-Cdc20 interaction is increased during mitosis, when the spindle checkpoint is active $[54,56,57]$. However, checkpoint inhibition of $\mathrm{APC} / \mathrm{C}^{\mathrm{Cdc} 20}$ turned out to be complex, involving more than the simple Mad2-Cdc20 interaction. In addition to Mad2, Mad3/BubR1-Bub3 binds to Cdc20 directly and inhibits APC/C. Furthermore, Bub1-Bub3 directly phosphorylates $\operatorname{Cdc} 20$ and inhibits $\mathrm{APC} / \mathrm{C}^{\mathrm{Cdc} 20}$. Although
Mad2 and Mad3/BubR1 can inhibit APC/CCdc20 independently, Mad3/BubR1 potentiates the ability of Mad2 to inhibit Cdc20 [50] and MCC containing BubR1/Mad3, Bub3, Mad2 and Cdc20 inhibits APC/C ${ }^{C d c 20}$ much more effectively than of Mad2 alone [15]. Furthermore, it has been recently shown that a complex containing Cdc20, BubR1/Mad3 and Mad2 accounts for most of the APC/C inhibitory activity in nocodazole-arrested HeLa cells [58]. Together, these results indicate that MCC is a major checkpoint inhibitor of APC/C. Nonetheless, the existence of MCC sub-complexes indicates that APC/CCdc20 inhibition by Mad2 and BubR1/Mad3 involves multiple, complex interactions.

\section{How does MCC inhibit APC/CCdc20?}

Because Cdc20 activates the ubiquitin ligase activity of $\mathrm{APC} / \mathrm{C}$ at least partially through substrate recruitment $[36,37,59]$, it was proposed that MCC interfered with $\mathrm{APC} / \mathrm{C}^{\mathrm{Cdc} 20}$ function by either blocking the access of substrates to Cdc20 or preventing the release of ubiquitinated substrates. Recent studies in budding yeast provide key insights into the mechanism by which MCC inhibits APC/ $\mathrm{C}$ and establish that the MCC subunit Mad3 (BubR1 in humans) blocks substrate access to $\mathrm{APC} / \mathrm{C}^{\mathrm{Cdc} 20}[60,61]$.

Many APC/C substrates contain short peptide motifs that mediate binding to and ubiquitination by $\mathrm{APC} / \mathrm{C}$, including the destruction box (D box) and the KEN box. Two groups have independently discovered that the budding yeast Mad3 protein contains one D box and two KEN boxes (Figure 1A) and that these degradation motifs of Mad3 are required for its spindle checkpoint function $[60,61]$. Furthermore, mutation of the N-terminal KEN box of Mad3 dramatically reduced the association of Mad3 with Cdc20 and Mad2 in vivo [61], indicating that the Mad3 KEN boxes are required for MCC formation. Although Mad3 binds directly to Cdc20 [60], this binding requires Mad2 in vivo $[60,61]$. Addition of Mad2 increases the Mad3-Cdc20 interaction about 4-fold in vitro [60]. How Mad2 stimulates Mad3 binding to Cdc20 remains unclear. Mad2 binding could conceivably alter the conformation of Cdc20, allowing more efficient Mad3 binding.

Because mutation of the N-terminal KEN box of Mad3 only reduced, but did not abolish, the Mad3-Cdc20 interaction, Burton and Solomon reasoned that other motifs of Mad3 ought to be involved in Cdc20 binding. Using an in vitro peptide competition assay, they determined that the KEN- and D-boxes of Mad3 bind to Cdc20 cooperatively. Furthermore, using the same assay, they showed that Mad3 competes with known Cdc20 substrates for Cdc20 binding, and this ability of Mad3 depends on its D- and KEN-boxes [60]. Taken together, these results indicate that Mad3 inhibits APC/C by blocking substrate binding to APC/CCdc20. 
A

\section{Mad3}

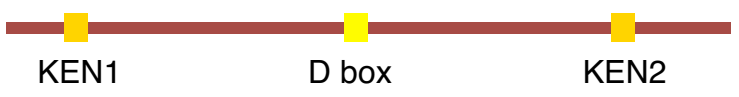

B

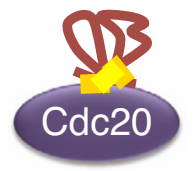

Monovalency

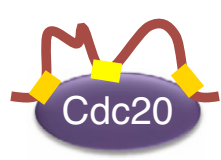

Multivalency

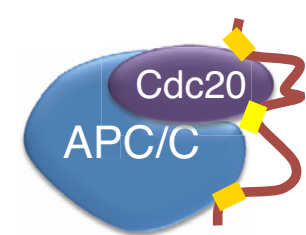

Shared-binding
Figure I

Mad3 and its interaction with Cdc20. A. Schematic drawing of Saccharomyces cerevisiae Mad3 (homolog of human BubRI). Mad3 contains several APC/C degradation motifs: a destruction box ( $D$ box) and two KEN boxes. $B$. Three possible mechanisms of Mad3 binding to Cdc20. The monovalency model proposes the cooperative binding of multiple motifs into a single docking site in $\mathrm{Cdc} 20$. The multivalency model proposes that the multiple degradation motifs of Mad3 bind to different docking sites in Cdc20. In the shared-binding model, the degradation motifs of Mad3 bind to different sites in both $\mathrm{Cdc} 20$ and APC/C.

Several non-exclusive models can explain the cooperative binding of KEN and D boxes of Mad3 to Cdc20 (Figure $1 \mathrm{~B})$. In the monovalency model, multiple APC/C degradation motifs of Mad3 simultaneously bind to a single substrate-binding site of Cdc20, thus blocking substratebinding by Cdc20. In the multivalency model, Cdc20 contains multiple substrate-binding sites. Cooperative binding of multiple KEN and D boxes of Mad3 to these sites blocks substrate binding by Cdc20. Finally, in the sharedbinding model, Mad3 inhibits $\mathrm{APC} / \mathrm{C}^{\mathrm{Cdc} 20}$ by binding to both Cdc20 and APC/C. This model takes into account the finding that APC/C can directly bind to destruction motifs $[62,63]$. Simultaneous binding of Mad3 to APC/C and Cdc20 would explain why mutations in the C-terminal KEN box of Mad3 have little effect on Mad3-Cdc20 binding and yet this KEN box is still required for the spindle checkpoint function of Mad3. High-resolution structural studies are required to understand the exact mode of interactions between Mad3 and Cdc20.

\section{Cdc20 ubiquitination and MCC disassembly}

In addition to inhibiting $\mathrm{APC} / \mathrm{C}^{\mathrm{Cdc}} 20$ by blocking substrate recruitment, Mad3 also destabilizes Cdc20 in a spindle checkpoint-dependent manner [64]. King et al. demonstrated that destabilization of Cdc20 requires the destruction motifs of Mad3 [61]. It has been recently shown that Cdc20 is ubiquitinated by APC/C when the spindle check- point is active $[65,66]$. Interestingly, Mad3 is unstable in G1 whereas it is stable in nocodazole-treated cells [61]. These results suggest the intriguing possibility that Mad3 binding to Cdc20 directs APC/C activity towards Cdc20, perhaps by mimicking substrate binding. Cdc20 ubiquitination leads to its proteasome-dependent degradation, ensuring that Cdc20 levels are kept below a certain threshold to prevent unscheduled APC/C activation [67].

Two recent studies have shown that degradation of Cdc20 is not the only outcome of Cdc20 ubiquitination $[65,66]$. Reddy et al. have shown that Cdc20 ubiquitination decreases its binding to Mad2 and to APC/C [66]. Cdc20 dissociation from Mad2 and $\mathrm{APC} / \mathrm{C}$ does not require the proteasome activity and Cdc20 degradation, as proteasome inhibitors did not block this dissociation. Furthermore, addition of the ubiquitin-conjugating enzyme UbcH10, but not its catalytically inactive mutant $\mathrm{UbcH} 10^{\mathrm{C} 114 \mathrm{~S}}$, increases both Cdc20 ubiquitination and the dissociation of Cdc20 from Mad2 and APC/C. Conversely, depletion of UbcH10 from HeLa cells decreases APC/C-mediated ubiquitination of Cdc20, stabilizes the Mad2-Cdc20 interaction, and delays anaphase initiation. This study thus establishes that Cdc20 ubiquitination by $\mathrm{APC} / \mathrm{C}$ is required for checkpoint inactivation and contributes to the dissociation of Cdc20 from Mad2 and possibly the disassembly of MCC[66]. In an accompanying study, Steigmeier $e t$ al. have identified the ubiquitin-specific protease USP44 as a spindle checkpoint component through an RNA interference (RNAi) screen $[65,66]$. Human cells depleted for USP44 by RNAi do not undergo mitotic arrest in the presence of spindle poisons. The checkpoint bypass of USP44 RNAi cells depends on APC/ C, although Mad2 and BubR1 (Mad3) localize normally to kinetochores. Thus, USP44 acts downstream of Mad2 in APC/C inhibition. Furthermore, USP44 antagonizes $\mathrm{APC} / \mathrm{C}$-mediated ubiquitination of Cdc20 in vivo. Recombinant USP44 directly deubiquitinates Cdc20 in vitro [65]. This study indicates that USP44 reduces Cdc20 autoubiquitination and protects the Mad2-Cdc20-containing checkpoint complexes from disassembly, although it remains to be determined whether USP44 also directly deubiquitinates mitotic APC/C substrates and prevents their degradation.

\section{The MCC paradox: inhibition through activation?}

These recent studies establish that, similar to APC/C substrates, Mad3 uses its APC/C degradation motifs to bind to Cdc20, thus blocking substrate binding to APC/CCdc20. Paradoxically, Mad3 binding to Cdc20 also activates the autoubiquitination of Cdc20, which has at least two functions: dissociation of Cdc20 from Mad2 and Cdc20 degradation by the proteasome. Thus, Mad3 binding to Cdc20 would trigger the dissociation of Cdc20 from Mad2. We 
propose a "dynamic MCC" model to reconcile these findings (Figure 2A). In this model, APC/C inhibition by the spindle checkpoint is achieved through a regulated, dynamic equilibrium of Cdc20 ubiquitination and deubiquitination. Checkpoint activation inhibits APC/CCdc20 by enhancing the formation of MCC and possibly its association with APC/C. Mad3 blocks access of substrates to APC/C $\mathrm{C}^{\mathrm{Cdc} 20}$ and simultaneously induces Cdc20 ubiquitination. In human cells, Cdc20 ubiquitination is stimulated by UbcH10 and possibly p31 ${ }^{\text {comet }}$ and is reversed by USP44. Cdc20 ubiquitination leads to Cdc20 dissociation from both APC/C and MCC components. Ubiquitinated $\mathrm{Cdc} 20$ is either degraded by the proteasome or deubiquitinated by USP44. If the spindle checkpoint stays on, MCC can re-assemble and bind to and inhibit the APC/C activity toward other substrates. Upon the proper attachment of the last chromosome to the mitotic spindle, the existing MCC can be rapidly disassembled through MCCand APC/C-dependent ubiquitination of Cdc20, leading to checkpoint inactivation.

The steady state levels of MCC are determined by the rates of MCC formation and disassembly. Three possible scenarios can explain the higher concentrations of MCC in mitosis. First, the rate of MCC formation increases during mitosis whereas the rate of MCC disassembly remains constant during the cell cycle. Second, an increase in the rate of MCC formation during mitosis is accompanied by a concomitant drop in the rate of MCC disassembly. The "dynamic MCC" model predicts a third scenario of MCC regulation, in which both rates of MCC formation and disassembly increase during mitosis (Figure 2B). By being dynamic, the levels of MCC are more responsive to the status of checkpoint signaling. Once the sister chromatids achieve proper attachment, MCC can be quickly inactivated to allow for APC/C activation and sister-chromatid separation. In contrast, the first two scenarios predict a slower and undesirable process of MCC inactivation and checkpoint silencing.

\section{Perspective}

Recent discoveries establish a mechanism for Mad3dependent inhibition of $\mathrm{APC} / \mathrm{C}^{\mathrm{Cdc} 20}$ and reveal the function and regulation of a dynamic Cdc20 ubiquitination/ deubiquitination cycle during mitosis. These studies support a "dynamic MCC" model for maintaining a robust spindle checkpoint and for the mechanistic coupling between APC/C inhibition and checkpoint inactivation. Many questions remain unresolved: does BubR1 (the vertebrate ortholog of Mad3) bind to Cdc20 and inhibit APC/CCdc20 using a mechanism similar to Mad3? Is USP44 conserved in other organisms? Is the activity of USP44 regulated by the spindle checkpoint? How are degradation of Cdc20 and its deubiquitination by USP44 coordinated? Future research aimed at addressing these questions will
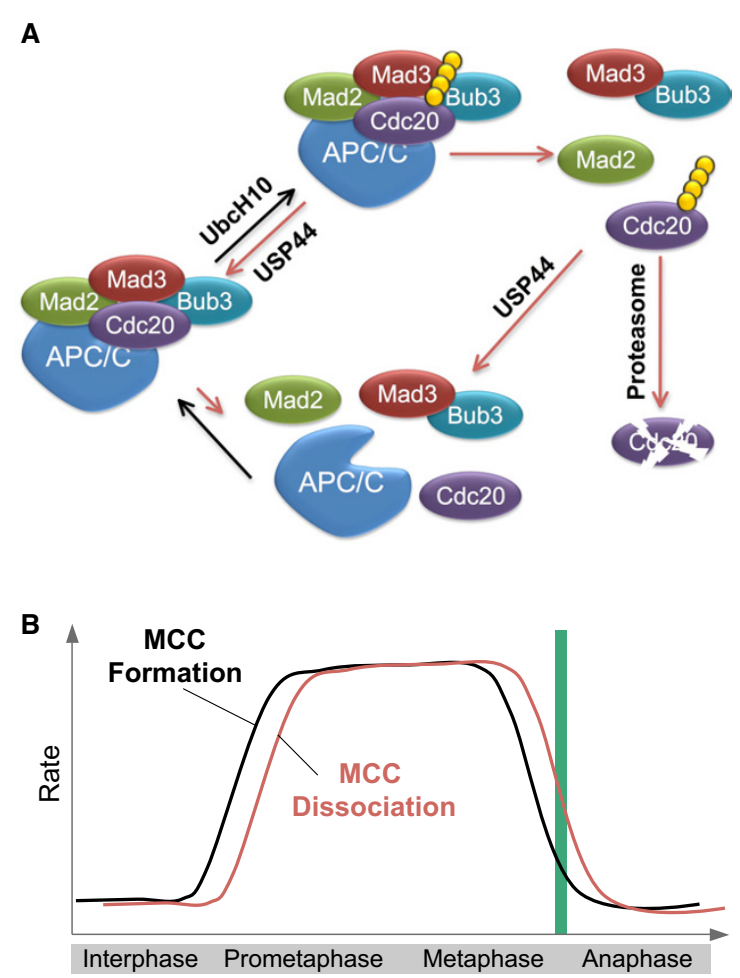

\section{Figure 2}

A dynamic model for MCC-mediated inhibition of APC/C. A. Mad3 uses its APC/C degradation motifs to bind to $\mathrm{Cdc} 20$ and blocks substrate binding of APC/CCdc20. Meanwhile, Mad3 binding to $\mathrm{Cdc} 20$ induces APC/C-dependent ubiquitination of $\mathrm{Cdc} 20$, which is antagonized by USP44. Ubiquitination of $\mathrm{Cdc} 20$ promotes the disassembly of MCC. Ubiquitinated $\mathrm{Cdc} 20$ is either degraded by the proteasome to reduce the cellular levels of $\mathrm{Cdc} 20$ or deubiquitinated by USP44. The deubiquitinated $\mathrm{Cdc} 20$ can be re-incorporated into MCC and associate with APC/C. Thus, upon checkpoint activation, a dynamic equilibrium of MCC formation and disassembly is achieved by the continuous cycles of ubiquitination and deubiquitination of $\mathrm{Cdc} 20$. This process directs the activity of $\mathrm{APC} / \mathrm{C}$ towards $\mathrm{Cdc} 20$ and reduces its activity towards cyclin B and securin. B. Schematic drawing of the rates of MCC formation and disassembly during mitosis. Upon checkpoint activation, Mad3 binds to $\mathrm{Cdc20}$ and inhibits APC/C, but Mad3 binding also induces Cdc20 ubiquitination and the disassembly of MCC. Thus, the rates of MCC formation and disassembly may both be enhanced during active spindle checkpoint signaling. An equilibrium is reached to keep the steady-state levels of MCC constant, analogous to a runner on a treadmill. This model is also consistent with the finding that, at any given time, only small pools of the Mad2, Cdc20, Bub3 and Mad3 molecules in a cell associate with APC/C. Once all sister chromatids achieve bi-orientation, the rate of MCC formation falls below that of MCC disassembly. The existing MCC complexes are rapidly disassembled, allowing the activation of APC/CCdc20 and checkpoint inactivation. 
further advance the molecular understanding of the spindle checkpoint.

\section{Competing interests}

The author(s) declare that they have no competing interests.

\section{Authors' contributions}

LADM and HY wrote the paper. All authors read and approved the final manuscript.

\section{Acknowledgements}

We thanks members of the Yu lab for helpful discussions. LADM also thanks DJ Clarke and JF Giménez-Abián for support and discussions. Research in our laboratory is supported in part by the National Institutes of Health (GM6I542).

\section{References}

I. Kops G], Weaver BA, Cleveland DW: On the road to cancer: aneuploidy and the mitotic checkpoint. Nat Rev Cancer 2005, 5:773-785.

2. Weaver BA, Cleveland DW: Does aneuploidy cause cancer? Curr Opin Cell Biol 2006, I 8:658-667.

3. Bharadwaj $\mathrm{R}, \mathrm{Yu} \mathrm{H}$ : The spindle checkpoint, aneuploidy, and cancer. Oncogene 2004, 23:2016-2027.

4. Rajagopalan H, Lengauer C: Aneuploidy and cancer. Nature 2004, 432:338-34l.

5. Peters JM: The anaphase promoting complex/cyclosome: a machine designed to destroy. Nat Rev Mol Cell Biol 2006, 7:644-656.

6. Yu H: Cdc20: A WD40 Activator for a Cell Cycle Degradation Machine. Mol Cell 2007, 27:3-16.

7. Rieder CL, Salmon ED: The vertebrate cell kinetochore and its roles during mitosis. Trends Cell Biol 1998, 8:310-318.

8. Maiato H, DeLuca J, Salmon ED, Earnshaw WC: The dynamic kinetochore-microtubule interface. I Cell Sci 2004, I I 7:546 I -5477.

9. Rieder CL, Khodjakov A: Mitosis and checkpoints that control progression through mitosis in vertebrate somatic cells. Prog Cell Cycle Res 1997, 3:301-312.

10. Rudner AD, Murray AW: The spindle assembly checkpoint. Curr Opin Cell Biol 1996, 8:773-780.

II. Zhou J, Yao J, Joshi HC: Attachment and tension in the spindle assembly checkpoint. J Cell Sci 2002, I I 5:3547-3555.

12. Pinsky BA, Biggins S: The spindle checkpoint: tension versus attachment. Trends Cell Biol 2005, I 5:486-493.

13. Rieder CL, Schultz A, Cole R, Sluder G: Anaphase onset in vertebrate somatic cells is controlled by a checkpoint that monitors sister kinetochore attachment to the spindle. J Cell Biol 1994, I 27:1301-1310.

14. Rieder CL, Khodjakov A, Paliulis LV, Fortier TM, Cole RW, Sluder G: Mitosis in vertebrate somatic cells with two spindles: implications for the metaphase/anaphase transition checkpoint and cleavage. Proc Natl Acad Sci USA 1997, 94:5I07-5I I 2.

15. Sudakin V, Chan GK, Yen TJ: Checkpoint inhibition of the APCI $C$ in HeLa cells is mediated by a complex of BUBRI, BUB3, CDC20, and MAD2. J Cell Biol 200I, I 54:925-936.

16. Luo X, Tang Z, Xia G, Wassmann K, Matsumoto T, Rizo J, Yu H: The Mad2 spindle checkpoint protein has two distinct natively folded states. Nat Struct Mol Biol 2004, I I:338-345.

17. De Antoni A, Pearson CG, Cimini D, Canman JC, Sala V, Nezi L, Mapelli M, Sironi L, Faretta M, Salmon ED, Musacchio A: The Mad I/ Mad2 complex as a template for Mad2 activation in the spindle assembly checkpoint. Curr Biol 2005, I 5:2 |4-225.

18. $\mathrm{Yu} \mathrm{H}$ : Structural activation of Mad2 in the mitotic spindle checkpoint: the two-state Mad2 model versus the Mad2 template model. I Cell Biol 2006, I73:153-157.

19. Musacchio A, Salmon ED: The spindle-assembly checkpoint in space and time. Nat Rev Mol Cell Biol 2007, 8:379-393.

20. Andrews CA, Diaz-Martinez LA, Gimenez-Abian JF, Guacci V, Vas $A C$, Clarke DJ: Evidence that the yeast spindle assembly checkpoint has a target other than the anaphase promoting complex. Cell Cycle 2005, 4:I555-I557.

21. Clarke DJ, Diaz-Martinez LA, Gimenez-Abian JF: Anaphase promoting complex or cyclosome? Cell Cycle 2005, 4:I585-I592.

22. Peters JM: The anaphase-promoting complex: proteolysis in mitosis and beyond. Mol Cell 2002, 9:931-943.

23. $\mathrm{Yu} \mathrm{H}$ : Regulation of APC-Cdc20 by the spindle checkpoint. Curr Opin Cell Biol 2002, I4:706-7I4.

24. Pines J: Mitosis: a matter of getting rid of the right protein at the right time. Trends Cell Biol 2006, 16:55-63.

25. Nasmyth K: How do so few control so many? Cell 2005, I 20:739-746.

26. Bashir T, Dorrello NV, Amador V, Guardavaccaro D, Pagano M: Control of the SCF(Skp2-CksI) ubiquitin ligase by the APC/ C(Cdh I) ubiquitin ligase. Nature 2004, 428: 190- I93.

27. Bashir T, Pagano M: Don't skip the GI phase: how APC/CCdh I keeps SCFSKP2 in check. Cell Cycle 2004, 3:850-852.

28. Machida YJ, Dutta A: The APC/C inhibitor, Emil, is essential for prevention of rereplication. Genes Dev 2007, 2 I : I 84- 194.

29. Sivaprasad U, Machida YJ, Dutta A: APC/C - the master controller of origin licensing? Cell Div 2007, 2:8.

30. Acquaviva C, Pines J: The anaphase-promoting complex/cyclosome: APC/C. J Cell Sci 2006, I 1 9:240 I-2404.

31. Baker DJ, Dawlaty MM, Galardy P, van Deursen JM: Mitotic regulation of the anaphase-promoting complex. Cell Mol Life Sci 2007, 64:589-600.

32. Vodermaier HC: APC/C and SCF: controlling each other and the cell cycle. Curr Biol 2004, I 4:R787-796.

33. Fang G, Yu H, Kirschner MW: Direct binding of CDC20 protein family members activates the anaphase-promoting complex in mitosis and GI. Mol Cell I998, 2:163-I7I.

34. Kramer ER, Gieffers C, Holzl G, Hengstschlager M, Peters JM: Activation of the human anaphase-promoting complex by proteins of the CDC20/Fizzy family. Curr Biol 1998, 8: I207-I210.

35. Burton JL, Solomon MJ: D box and KEN box motifs in budding yeast Hsl I p are required for APC-mediated degradation and direct binding to Cdc20p and CdhIp. Genes Dev 200I, I 5:2381-2395.

36. Hilioti Z, Chung YS, Mochizuki Y, Hardy CF, Cohen-Fix O: The anaphase inhibitor PdsI binds to the APC/C-associated protein Cdc20 in a destruction box-dependent manner. Curr Biol 200 I, I I:|347-I352.

37. Pfleger CM, Lee E, Kirschner MW: Substrate recognition by the Cdc20 and CdhI components of the anaphase-promoting complex. Genes Dev 200I, I 5:2396-2407.

38. Schwab M, Neutzner M, Mocker D, Seufert W: Yeast Hct I recognizes the mitotic cyclin Clb2 and other substrates of the ubiquitin ligase APC. Embo J 200I, 20:5I65-5I75.

39. Eytan E, Moshe Y, Braunstein I, Hershko A: Roles of the anaphasepromoting complex/cyclosome and of its activator Cdc20 in functional substrate binding. Proc Natl Acad Sci USA 2006, 1 03:208I-2086.

40. Shteinberg M, Protopopov Y, Listovsky T, Brandeis M, Hershko A: Phosphorylation of the cyclosome is required for its stimulation by Fizzy/cdc20. Biochem Biophys Res Commun 1999, 260:193-198.

41. Golan A, Yudkovsky Y, Hershko A: The cyclin-ubiquitin ligase activity of cyclosome/APC is jointly activated by protein kinases Cdkl-cyclin B and Plk. J Biol Chem 2002, 277: I5552-15557.

42. Herzog F, Mechtler K, Peters JM: Identification of cell cycledependent phosphorylation sites on the anaphase-promoting complex/cyclosome by mass spectrometry. Methods Enzymol 2005, 398:23 I-245.

43. Kraft C, Herzog F, Gieffers C, Mechtler K, Hagting A, Pines J, Peters JM: Mitotic regulation of the human anaphase-promoting complex by phosphorylation. Embo J 2003, 22:6598-6609.

44. Yudkovsky Y, Shteinberg M, Listovsky T, Brandeis M, Hershko A: Phosphorylation of Cdc20/fizzy negatively regulates the mammalian cyclosome/APC in the mitotic checkpoint. Biochem Biophys Res Commun 2000, 27 I:299-304.

45. Chung E, Chen $\mathrm{RH}$ : Phosphorylation of $\mathrm{Cdc20}$ is required for its inhibition by the spindle checkpoint. Nat Cell Biol 2003, 5:748-753. 
46. Tang Z, Shu H, Oncel D, Chen S, Yu H: Phosphorylation of Cdc20 by Bubl provides a catalytic mechanism for APC/C inhibition by the spindle checkpoint. Mol Cell 2004, 16:387-397.

47. Hall MC, Warren EN, Borchers $\mathrm{CH}$ : Multi-kinase phosphorylation of the APC/C activator CdhI revealed by mass spectrometry. Cell Cycle 2004, 3: I278-1284.

48. Eckerdt F, Strebhardt K: Polo-like kinase I: target and regulator of anaphase-promoting complex/cyclosome-dependent proteolysis. Cancer Res 2006, 66:6895-6898.

49. Tang Z, Bharadwaj R, Li B, Yu H: Mad2-Independent inhibition of APCCdc20 by the mitotic checkpoint protein BubRI. Dev Cell 200I, I:227-237.

50. Fang G: Checkpoint protein BubRI acts synergistically with Mad2 to inhibit anaphase-promoting complex. Mol Biol Cell 2002, I 3:755-766.

5I. Reimann JD, Freed E, Hsu JY, Kramer ER, Peters JM, Jackson PK: Emil is a mitotic regulator that interacts with Cdc20 and inhibits the anaphase promoting complex. Cell 200I, 105:645-655.

52. Reimann JD, Gardner BE, Margottin-Goguet F, Jackson PK: Emil regulates the anaphase-promoting complex by a different mechanism than Mad2 proteins. Genes Dev 200 I, I 5:3278-3285.

53. Li Y, Gorbea C, Mahaffey D, Rechsteiner M, Benezra R: MAD2 associates with the cyclosome/anaphase-promoting complex and inhibits its activity. Proc Natl Acad Sci USA 1997, 94: I243 I-I 2436.

54. Fang $\mathrm{G}, \mathrm{Yu} \mathrm{H}$, Kirschner MW: The checkpoint protein MAD2 and the mitotic regulator CDC20 form a ternary complex with the anaphase-promoting complex to control anaphase initiation. Genes Dev 1998, I 2:187/-I883.

55. Hwang LH, Lau LF, Smith DL, Mistrot CA, Hardwick KG, Hwang ES, Amon A, Murray AW: Budding yeast Cdc20: a target of the spindle checkpoint. Science 1998, 279:104I-1044.

56. Kallio M, Weinstein J, Daum JR, Burke DJ, Gorbsky GJ: Mammalian p55CDC mediates association of the spindle checkpoint protein Mad2 with the cyclosome/anaphase-promoting complex, and is involved in regulating anaphase onset and late mitotic events. / Cell Biol 1998, I41:1393-1406.

57. Wassmann K, Benezra R: Mad2 transiently associates with an APC/p55Cdc complex during mitosis. Proc Natl Acad Sci USA 1998, 95:11193-11198.

58. Braunstein I, Miniowitz S, Moshe Y, Hershko A: Inhibitory factors associated with anaphase-promoting complex/cylosome in mitotic checkpoint. Proc Natl Acad Sci USA 2007, 104:4870-4875.

59. Visintin R, Prinz S, Amon A: CDC20 and CDHI: a family of substrate-specific activators of APC-dependent proteolysis. Science 1997, 278:460-463.

60. Burton JL, Solomon MJ: Mad3p, a pseudosubstrate inhibitor of APCCdc20 in the spindle assembly checkpoint. Genes Dev 2007, 21:655-667.

61. King EM, van der Sar SJ, Hardwick KG: Mad3 KEN Boxes Mediate both Cdc20 and Mad3 Turnover, and Are Critical for the Spindle Checkpoint. PLoS ONE 2007, 2:e342.

62. Meyn MA 3rd, Melloy PG, Li J, Holloway SL: The destruction box of the cyclin Clb2 binds the anaphase-promoting complex cyclosome subunit Cdc23. Arch Biochem Biophys 2002, 407:189-195.

63. Carroll CW, Enquist-Newman M, Morgan DO: The APC subunit Docl promotes recognition of the substrate destruction box. Curr Biol 2005, 15: I - I8.

64. Pan J, Chen RH: Spindle checkpoint regulates Cdc20p stability in Saccharomyces cerevisiae. Genes Dev 2004, I8: |439-|45|.

65. Stegmeier F, Rape M, Draviam VM, Nalepa G, Sowa ME, Ang XL, McDonald ER 3rd, Li MZ, Hannon GJ, Sorger PK, et al.: Anaphase initiation is regulated by antagonistic ubiquitination and deubiquitination activities. Nature 2007, 446:876-88I.

66. Reddy SK, Rape M, Margansky WA, Kirschner MW: Ubiquitination by the anaphase-promoting complex drives spindle checkpoint inactivation. Nature 2007, 446:921-925.

67. Chen RH: Dual inhibition of Cdc20 by the spindle checkpoint. J Biomed Sci 2007, I 4:475-479.

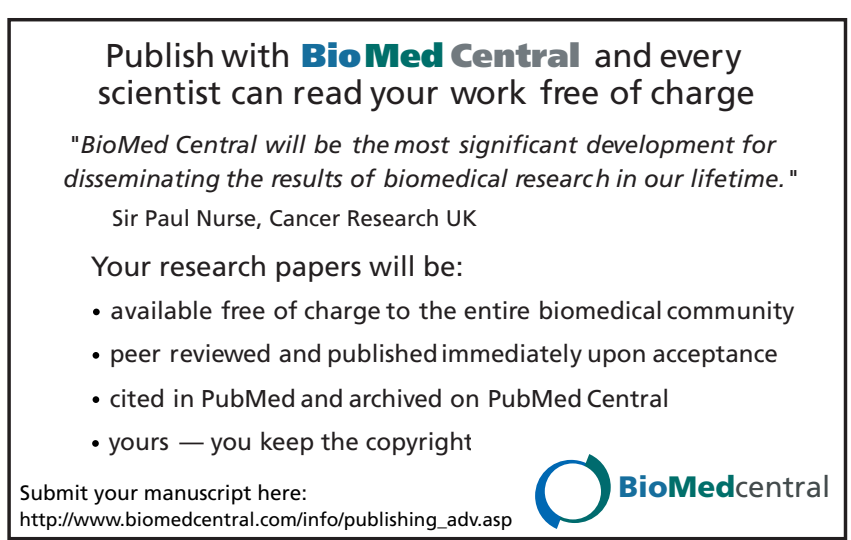

